\title{
Calibration of Force Sensitive Resistor used in Force Controlled Grippers
}

\author{
Gottipati S Lakshmi \\ (PG student) \\ Department of Electrical Engineering(C\&I) \\ UVCE, Bangalore university \\ Bangalore, Karnataka, India
}

\author{
Hemavathi R \\ (Assoc. Professor) \\ Department of Electrical Engineering(C\&I) \\ UVCE, Bangalore university \\ Bangalore, Karnataka, India
}

\begin{abstract}
This paper aims to present the calibration of a piezoresistive force sensitive sensors for the usage in gripping devices.A Force Sensitive Resistor(FSR) sensor is a device that allow measuring static and dynamic forces applied to contact surface.Their range of responses is basically depending on the variation of its electrical resistance. Studies have shown that the FSR sensors are usually applied for robotic grippers and for biomedical fields. This paper provides a brief overview of the application of FSR sensor as a feedback element for force controlled gripper.The used calibration technique is presented in parallel with the hardware and software elements. The results revealed that the FSR sensors are very useful devices that are able to provide a good contact force control, particularly for gripping.
\end{abstract}

Keywords-Piezo resistive, Force Sensitive Resistor(FSR), contact force, calibration, Arduino UNO,

\section{INTRODUCTION}

In the applications of automation, namely gripping devices the end effectors are to be able to meet all the given tasks, or to understand the variations of the surrounding environment, specific sensors, actuators and algorithms are required. Some of the end effectors are anthropomorphic or nonanthromorphic grippers, used to substitute human movements and operations.

From a mechanical point of view, the gripping systems require at least two contact points between the gripper and the object. Various studies considered the development and the control of the grip forces, by monitoring of various sensing information, similar to those of a human hand, for the usage in areas such as industry, research, military and biomedical applications.

The tactile sensing is strategic for the implementation of a safe control of grippers interacting with humans, objects, and possibly in unstructured environments, where usually interaction is expected or planned at a specific locations (typically at the end-effector tip), force/torque sensors have been widely and successfully adopted. On the other hand, a better capability of controlling is required. The better controlling capability is needed, for example where the location and the characteristics of the contact could not be exhaustively predicted or possibly modeled a priori. Skin-like sensors and appropriate sensing methods for the processing of distributed tactile information are needed, in order to enable the implementation of safe interaction strategies and real-time system response.
The tactile sensors provide different information through the physical interaction with the surrounding environment [14]. They must be incorporated into skin surfaces with compliance, for conforming locally to surfaces, and with adequate friction for handling objects securely. The sensors and the synthetic elastomeric skin must also be robust enough to survive repeated impacts, controlled or uncontrolled touch forces, as well as abrasions. Force sensitive resistor is considered, regarding the interaction between the gripper and the surrounding environment.

The interactions like manipulation, grasp force control, surface contact locations, kinematics, and stability appraisal are taken into account for a stable handling, friction and hardness and real time reaction to environmental factors is required.

Taking into account these problems, we reviewed the characteristics of different tactile sensors. One possible tactile sensing solution could be based on the force sensing resistors, based on the piezo resistive principle. Before the integration of these sensors, several calibrations and laboratory analysis are required. The force sensitive resistors were calibrated using the digital force meter, commercially available electronic development board and a custom made software platform.

The experiments provided sufficient extended information regarding the functionality of the sensors, and will allow us to extend the research towards integration of these sensors on grippers.

In Section 2, voltage divider circuit of Force Sensitive Resistor(FSR) is summarized. In Section 3, the calibration methodology of FSR is presented. Results and discussion are presented in Section 4.

\section{VOLTAGE DIVIDER CIRCUIT OF FORCE SENSITIVE RESISTOR}

\section{A. piezo resistive Force sensitive resistors}

Piezo-resistive sensors change their internal electrical resistance under applied pressure on the active surface. They are of interest, because of low weight, flexibility, small response time (under $3 \mu$ s response time) and variable resistor functionality. The force sensing range is between 0.1 Newton and 100 Newton, with an electrical resistance ranging from $10 \mathrm{M}$ Ohms $(\mathrm{M} \Omega)$ without any load, down to $100 \mathrm{Ohms}(\Omega)$ at maximum load applied on the sensitive surface.

The main advantages of FSRs are: thin size, verygood shock resistance, low power requirement, fast response to force 
changes, robustness against noise, simple conditioning circuits, ability to fabricate using flexible materials, and low unit cost compared to other commercial force sensors. However, these sensors have some disadvantages that need to be compensated for, such as non-linear behavior and repeatability error [3].

The most basic Force Sensing Resistors (FSR) consists of two membranes separated by a thin air gap. The air gap is maintained by a spacer (Fig.2. Spacer Adhesive) mounted around the edges of the membrane and by the rigidity of the two membranes.

One of the membranes has two sets of inter digital contacts (Fig.2. Active sensing area) that are electrically distinct, with each set connecting to one trace on a tail. The other membrane is coated with FSR ink (Fig.1. Force sensitive layer, printed with carbon based ink). When pressed or touched, the FSR ink carbon based structures act as a short between the conductive traces from the contact area, resulting in a resistance that depends on the applied force.

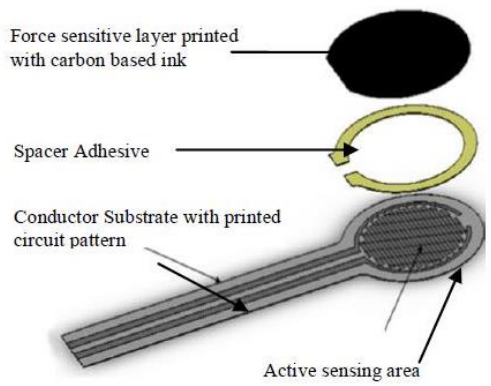

Figure-1:Interlink Electronics FSR 400 sensor

When the two substrates are pressed together, the microscopic protrusions on the FSR ink surface short across the interdigital fingers of the facing surface. At low forces, only the tallest protrusions make contact, while at higher forces, there are more and more contact points between the two substrates. The result is that the resistance between the electro conductive traces is inversely proportional to the applied force.

At low forces, only the tallest protrusions make contact, while at higher forces, there are more and more contact points between the two substrates. The result is that the resistance between the electro conductive traces is inversely proportional to the applied force.

Note that FSR sensors show the great potential for various application, including robotic fingers and grippers despite some drawbacks were found.

\section{B. Voltage divider circuit}

The FSR sensors exhibits resistance change in correspondence to the applied force. Therefore, avoltage divider circuit as shown in figure was used to convert the resistance changes to voltage values to be acquired by microcontroller. The basic voltage divider provides a $0 \div+5 \mathrm{~V}$ Dc voltage range for each FSR, according to the applied force and the measured resistor $(10 \mathrm{k} \Omega)$. The voltage divider circuit of a FSR is shown in figure(2).

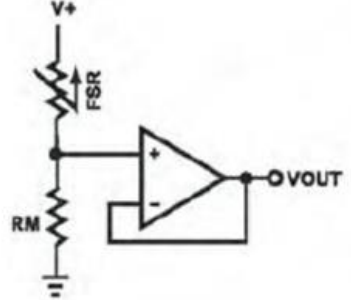

Figure-2: voltage divider circuit of FSR

Voltage drop across FSR $=(\mathrm{V}+)-$ Vout

Voltage drop across $\mathrm{Rm}=$ Vout

Applying ohm's law

$$
\begin{gathered}
(\mathrm{V}+)-(\text { Vout })=\mathrm{Rfsr} * \mathrm{I} \\
\text { Vout=Rm*I }
\end{gathered}
$$

From equations (1)\& (2)

$$
\begin{aligned}
& ((\mathrm{V}+)-\text { Vout }) / \mathrm{Rfsr}=\mathrm{Vout} / \mathrm{Rm} \\
& \mathrm{V}+/ \text { Vout }=(\mathrm{Rm}+\mathrm{Rfsr}) / \mathrm{Rm} \\
& \text { Vout=Rm* }(\mathrm{V}+) /(\mathrm{Rm}+\mathrm{Rfsr}) \\
& \mathrm{Rfsr}=\left((\mathrm{V}+)^{*} \mathrm{Rm}\right) / \text { Vout }
\end{aligned}
$$

For simple force-to-voltage conversion, the FSR device is tied to a measuring resistor in a voltage divider (see figure below) and the output is described by the following equation.

$$
V_{\text {OUT }}=\frac{R_{M} V+}{\left(R_{M}+R_{F S R}\right)}
$$

In the configuration shown, the output voltage increases with increasing force. If FSR and RM are swapped, the output swing will decrease with increasing force.In the configuration shown, the output voltage increases with increasing force. If RFSR and RM are swapped, the output swing will decrease with increasing force. The measuring resistor, RM, is chosen to maximize the desired force sensitivity range and to limit current. Depending on the impedance requirements of the measuring circuit, the voltage divider could be followed by an op-amp. A family of force vs. VOUT curves is shown on the graph below for a standard FSR in a voltage divider configuration with various $\mathrm{RM}$ resistors. A V+ of $5 \mathrm{~V}$ was used for these examples as shown in figure. The relationship between the force and the resistance is shown in figure. When force increases, the resistance decreases non-linearly.

\section{CALIBRATION METHODOLOGY}

An experiment is conducted for the calibration of the FSR sensor by using a digital force meter, FSR sensor (400 series by Interlink Electronics), arduino Uno micro controller. To interface a force sensor with Arduino uno, we are going use 8 bit ADC (Analog to Digital Conversion) feature in arduno uno. FSR400 is one of the cheapest force sensors in the market[6-7]. The features are actuation force as low as $0.2 \mathrm{~N}$ and sensitivity range to $30 \mathrm{~N}$. Force Sensing Resistors, or FSR's, are robust polymer thick film (PTF) devices that exhibit a decrease in resistance with increase in force applied to the surface of the sensor. This force sensitivity is optimized for use in human machine interface devices including automotive electronics, medical systems, industrial controls and robotics. So we can apply only $1 \mathrm{Kg}$ of weight. If weights 
higher than $1 \mathrm{Kg}$ applied the sensor might show some deviations. If it's increased more than $3 \mathrm{Kg}$. the sensor might damage permanently.

An experiment is conducted by a wooden block of diameter $0.9 \mathrm{~mm}$ was placed over FSR sensor. This was done so that the force applied on the FSR is uniformly distributed and the circuit was completed by connecting the FSR to Arduino uno microcontroller. FSR was fixed on the platform of the force meter. Now the force meter was used to apply force gradually and corresponding digital values were recorded manually. While increasing the load on the FSR by force meter, the digital values of FSR was also increasing. The load on the force meter was applied in range of $(0-25 \mathrm{~N})$ and the FSR reading varied from $0-1023$. The connection of force sensor to the arduino uno board is shown in below figure(3).

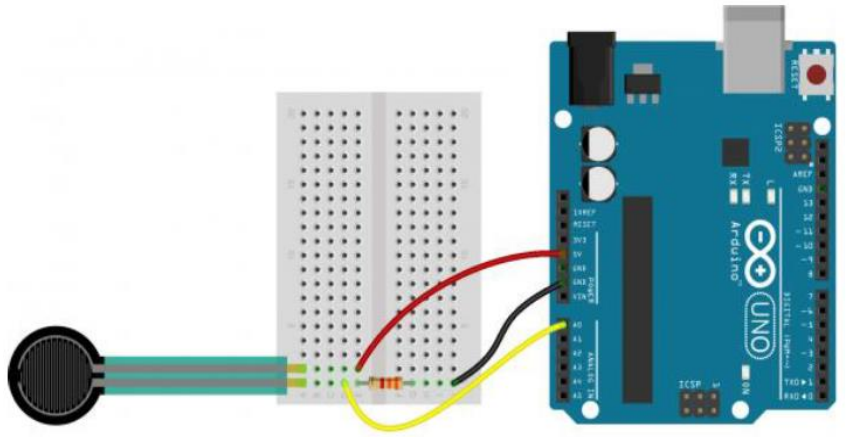

Figure(3): Arduino connection of FSR

\section{RESULTS AND DISCUSSION}

This section illustrates and discuss the results obtained from the calibration and characterization tests. The relationship derived between the digital output of FSR sensor and the value of force(weights in Newtons) is shown in below figure(4). our setup RM The figures(5-6) shows the relationship between the applied force in newtons and the resistance in kilo ohms and the voltage in volts. In value was taken to be $10 \mathrm{k} \Omega$. From the below figures we observed that the increase in force will reduce the resistance of FSR and increases the voltage. It is observed that the motor of actuator rotates according to the range of the digital value of Force Sensitive Resistor(FSR). In addition if the constant weights was kept for a longer time(a few minutes), the sensor voltage stabilized to a steady value.

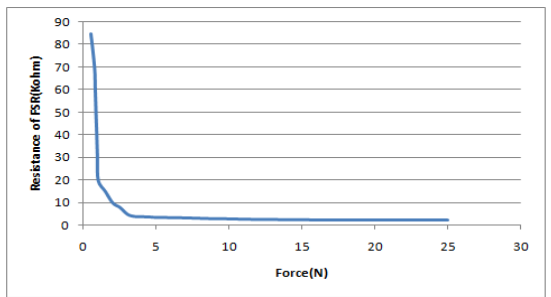

Figure-4:plot of digital values of FSR vs Force(N)

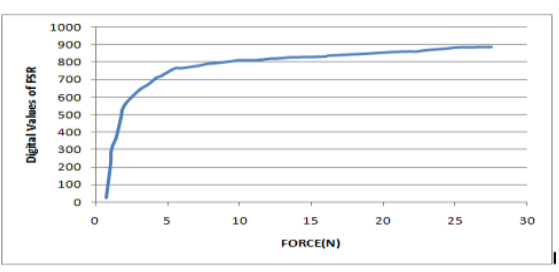

Figure-5: plot of Force(N) vs resistance of FSR(kQ)

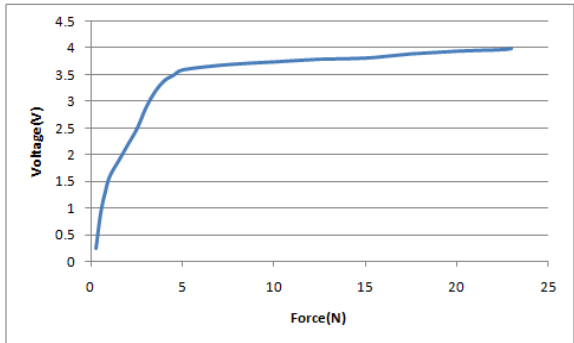

Figure-6: plot of Force(N)Vs Voltage(V)

\section{CONCLUSION}

In this paper we demonstrated the brief overview of the use of Force Sensitive Resistor(FSR). The results shows the FSR sensors are sufficient and reliable devices to measure and record the detected force. The results showed that there was a significant difference of data measurement produced by FSR sensor at different hardware applications. It is observed that the calibrations are required before starting the experiment to ensure the repeatability and reliability of the acquired data.

Moreover, the FSR sensors tend to produce a non-linear relationship within the $0 \mathrm{~N}$ to $4 \mathrm{~N}$ force range. As such, some difficulties may occur in measuring data for a higher force range test. Future studies could monitor the substructure wear of the sensors, after different and prolonged usage, allowing us to estimate the applied force/ voltage/ internal electrical resistance variation over time, as a response to permanent usage on grippers

\section{ACKNOWLEDGMENT}

The authors wish to thank the Faculty of Electrical Engineering, University visvesvaraya college of Engineering, Bangalore university for providing a platform to carry out the research activities

\section{REFERENCES}

[1] Alexandru BARNEA, Cezar OPRISAN, Dumitru OLARU,"Force sensitive resistors calibration for the usage in gripping devices" The 3rd International Conference on diagnosis and prediction in mechanical engineering systems May 31 - June 1, 2012, Gala ti, Romania

[2] C. Lebosse, B. Bayle, M. De Mathelin, and P. Renaud, "Nonlinear modeling of low cost force sensors," 2008 IEEE Int. Conf. Robot. Autom., pp. 3437-3442, 2008. Q. Wang, H. Zheng, "Route and spectrum selection in dynamic spectrum networks," in Proc. IEEE CCNC 2006, pp. 625-629, Feb. 2006.

[3] J. a. Flórez and a. Velásquez, "Calibration of force sensing resistors (fsr) for static and dynamic applications," 2010 IEEE ANDESCON Conf. Proceedings, ANDESCON 2010, pp. 2-7, 2010H. Khalife, N. Malouch, S. Fdida, "Multihop cognitive radio networks: to route or not to route," IEEE Network, vol. 23, no. 4, pp. 20-25, 2009.

[4] A. Hollinger and M. M. Wanderley, "Evaluation of Commercial ForceSensing Resistors," pp. 1-4, 2006

[5] M. W. Gilbertson and B. W. Anthony, "Ergonomic control strategies for a handheld force-controlled ultrasound probe," 2012 IEEE/RSJ International Conference on Intelligent Robots and Systems, Vilamoura, 2012, pp. 1284-1291, doi: 10.1109/IROS.2012.6385996

[6] Interlink Electronics FSR ${ }^{4} 400$ Series Data Sheet

[7] Force Sensitive Resistor Hookup Guide - learn.sparkfun.com [Internet]. Learn.sparkfun.com. 2020 [cited 26 June 2020]. Available from: https://learn.sparkfun.com/tutorials/force-sensitive-resistor-hookupguide/all 